\title{
A Combined Experimental and Modelling Approach to Elastic-Plastic Crack Driving Force Calculation in the Presence of Residual Stresses
}

\author{
H.E. Coules ${ }^{1}$ • D.J. Smith ${ }^{1}$ P.J. Orrock ${ }^{1}$ - K. Abburi Venkata ${ }^{1}$ - T. Pirling ${ }^{2}$
}

Received: 15 March 2016 / Accepted: 9 May 2016 / Published online: 19 May 2016

(C) The Author(s) 2016. This article is published with open access at Springerlink.com

\begin{abstract}
Since all residual stress measurement methods have inherent limitations, it is normally impractical to completely characterise a three-dimensional residual stress field by experimental means. This lack of complete information makes it difficult to incorporate measured residual stress data into the analysis of elastic-plastic fracture without resorting to simplified methods such as the Failure Assessment Diagram (FAD) approach. We propose a technique in which the complete residual stress field is reconstructed from measurements and used in finite element analysis of the fracture process. Residual elastic strains and stresses in three-point bend fracture specimens were measured using neutron diffraction and an iterative method was used to generate a self-consistent estimate of the complete residual stress field. This enabled calculation of the $J$ contour integral for a specimen acted on by both residual stress and an externally-applied load, allowing the interaction between residual and applied stress to be observed in detail.
\end{abstract}

Keywords Residual stress $\cdot$ Reconstruction $\cdot$ Fracture · Neutron diffraction $\cdot J$-integral

H. E. Coules

harry.coules@bristol.ac.uk

1 Department of Mechanical Engineering, University of Bristol, Bristol BS8 1TR, UK

2 Institut Max von Laue - Paul Langevin, 6 rue Jules Horowitz, BP156, F-38042 Grenoble, France

\section{Introduction}

Residual and thermal stresses can influence both brittle fracture and elastic-plastic fracture. Within linear elastic fracture mechanics, the effect of internally self-equilibrating stresses on the crack driving force can be understood in much the same way as the effect of external loading. Under the superposition principle due to Bueckner [1], the singular component of the stress field at a crack tip caused by the relaxation of stresses during introduction of the crack is identical to that caused by equivalent loading applied to the crack faces. In the case of elastic-plastic fracture however, plastic deformation of material surrounding the crack tip can cause the internally selfequilibrating component of the stress field to change prior to fracture initiation. Consequently, the effects of thermal or residual stresses combine with the action of externally-applied loads in a non-linear manner [2].

This complicates the prediction of fracture initiation and crack growth. In structural integrity assessment procedures such as R6 Rev. 4 maintained by EDF Energy and others [3, 4], and the British standard BS 7910:2013 [5], combinations of residual and applied loading can be accounted for using an interaction factor (denoted $V$ ) to adjust the apparent contribution of residual stress loading to the stress intensity factor at a given crack. During an assessment, this factor is applied to the stress intensity factor calculated for secondary (i.e. self-equilibrating) stresses before it is combined with the corresponding stress intensity factor for primary (i.e. externally-applied) loading. In general, secondary stresses have a greater influence on fracture at low levels of primary loading [6]. At higher primary load levels, pre-existing residual stresses tend to be partly relaxed by plastic deformation prior to fracture and the formulation of $V$ within R6 reflects this.

More generally, energy-based criteria are often used to predict elastic-plastic fracture initiation and the presence of 
residual stress changes the strain energy release rate at a crack. When the $J$ contour integral is used as a fracture initiation criterion in the presence of thermal and residual stresses, it must be formulated to include terms which would otherwise be equal to zero. For the case of thermal stress [7]:

$J=\int_{\Gamma}\left(W \delta_{1 i}-\sigma_{i j} \frac{\partial u_{j}}{\partial x_{1}}\right) n_{i} d s+\int_{A} \sigma_{i i} \frac{\partial \varepsilon_{i j}^{t h}}{\partial x_{1}} d A$

where $\Gamma$ is a closed contour surrounding the crack tip for which $n_{i}$ is an outward-facing normal vector and $A$ is the internal area. $\sigma_{i j}$ is the stress tensor, $W$ the strain energy density, and $\varepsilon_{i j}^{\text {th }}$ the thermal strain tensor. $x_{i}$ and $u_{i}$ are the position and displacement vectors respectively, and $\delta_{i j}$ is the Kronecker delta. As is the case in the absence of thermal/residual stresses, the $J$-integral is only equal to the strain energy release rate for ideal non-linear elastic materials; its application to real elastic-plastic materials via models based on incremental plasticity is approximate. Lei proposed a similar expression for $J$ in the presence of residual stress [8], closely following a derivation due to Wilson and Yu for the case of thermal stress [9]:

$$
J=\int_{\Gamma}\left(W \delta_{1 i}-\sigma_{i j} \frac{\partial u_{j}}{\partial x_{1}}\right) n_{i} d s+\int_{A}\left(\sigma_{i j} \frac{\partial \varepsilon_{i j}}{\partial x_{1}}-\frac{\partial W}{\partial x_{1}}\right) d A
$$

where the total strain $\varepsilon_{i j}$ is the sum of all initial, elastic and plastic strains. Using this expression, $J$ can be estimated so long as the residual stress, total strain, and strain energy density fields in the cracked body are known. To determine these it is normally necessary to simulate the process by which the residual stress field is formed, the introduction of a crack into the residual stress field, and any subsequent loading by externally-applied forces. Models of this nature typically require experimental validation; particularly for residual stresses formed via complex thermo-mechanical processes such as welding $[4,10]$. Alternatively, when residual stresses are measured from a physical specimen it is not normally possible to calculate the $J$-integral explicitly. Firstly, due to the difficulty involved in residual stress field characterisation in metals any measured stress field data tends to be insufficiently complete for $J$-integral calculation. Secondly, the residual stress field alone insufficient for calculation of the $J$-integral; distributions of total strain and strain energy density, including energy dissipated as plastic work for real elastic-plastic materials, are also needed.

In this study we propose a combined experimental/ analytical treatment of elastic-plastic fracture in the presence of residual stress. The residual stress and elastic strain fields which exist in a specimen before and after the introduction of a defect are reconstructed from point-wise data measured using neutron diffraction. Using this information along with modelling of the residual stress introduction and fracture loading of the specimen, elastic and elastic-plastic fracture parameters are evaluated explicitly. In this way, the effect of an experimentally-measured residual stress field on elasticplastic fracture can be analysed without resorting to simplified methods such as the R6 $V$ factor to account for the interaction between residual and applied loading.

\section{Experiments}

\section{Overview}

Rectangular bar specimens of high-strength aluminium alloy were prepared and approximately half of these were plastically indented to produce a nominally identical residual stress field in each one. Wire Electric Discharge Machining (EDM) was used to produce crack-like notches of predetermined length into most of these specimens. The residual stress field in notched and un-notched specimens was measured using neutron diffraction. Notched specimens were then subjected to three-point bend loading to determine the difference in apparent fracture toughness between plain and residually-stressed specimens. The compression, notch cutting and three-point bend loading operations were simulated using the finite element method, and fracture parameters were determined from the resulting stress and strain fields. Finally, a technique for calculating fracture parameters based on the measured residual stress field using the finite element method was implemented, and fracture parameters calculated using different techniques were compared.

\section{Specimens}

Nineteen oblong pieces of wrought aluminium alloy 7075-T6 were manufactured with dimensions $150 \times 30 \times 15 \mathrm{~mm}$, as shown in Fig. 1. For all of the specimens the material's rolling direction was parallel with the $30 \mathrm{~mm}$ edge, producing a fracture specimen in the T-L orientation. Approximately half of these specimens were indented on both sides using a pair of cylindrical punches at the location shown in Fig. 1, allowing a residual stress field to be produced within the specimen in a repeatable manner [11]. The compression process was designed to produce residual stresses which would cause strong opening-mode loading for a notch of $15 \mathrm{~mm}$ length. The compression tool faces were made from BS970-1:1983 817 M40 (EN24) tool steel heat-treated to a hardness of approximately $470 \mathrm{HV}$, and were located on the specimen using a speciallymade jig. Specimens were compressed with a force of $75 \mathrm{kN}$ normal to the specimen's surface ramped over $60 \mathrm{~s}$, which resulted in a reduction in thickness of approximately $1.4 \mathrm{~mm}$ in the indented region. Ten specimens were subjected to this compression operation, while nine were left in an un-punched state. 
Fig. 1 Geometry of the threepoint-bend fracture specimens

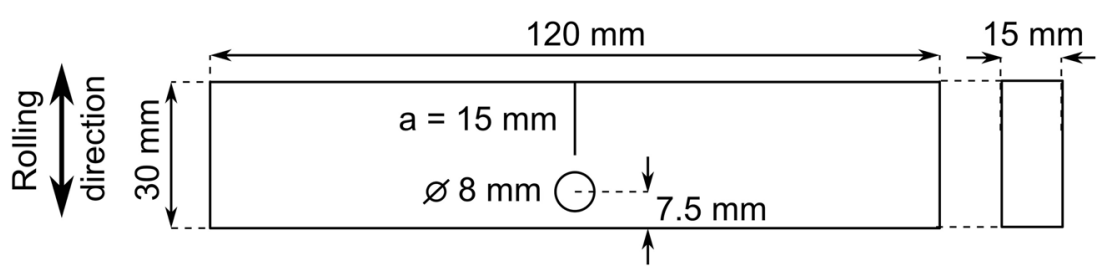

Notches were cut in the specimens to predetermined depths using wire EDM. A summary of the different specimens produced is shown in Table 1. The tip at the end of the EDM-cut notch was approximately semi-circular with a radius of 83 $\pm 3 \mu \mathrm{m}$ for all specimens. Uniaxial tensile tests were performed on cylindrical specimens from the same batch of material to determine its mechanical properties (see Appendix A).

\section{Neutron Diffraction Measurements}

Neutron diffraction was used to measure residual elastic strain in three indented specimens: one with no notch, one with a $7.5 \mathrm{~mm}$ notch and one with a $15 \mathrm{~mm}$ notch. The specimens were not externally loaded during measurement and the measurements were carried out using the SALSA monochromatic diffractometer [12] (Institut Laue Langevin, France).

In neutron diffraction strain scanning, Bragg's law is used to determine the inter-planar spacing within crystalline material from the distribution of scattered neutrons $[13,14]$. The technique is suitable for polycrystalline materials and relies on there being a relatively large number of material grains in the neutron scattering volume. The lattice spacing can be determined as a function of orientation and position within the specimen by moving the scattering volume. By comparing lattice spacings measured in stressed and un-stressed material, the elastic strain and hence the stress within a specimen can be found. In this experiment, an incident neutron wavelength of $1.644 \AA$ was used, enabling scattering angles for the measured reflections of $2 \theta \approx 90^{\circ}$. The mean grain size of the material was approximately $15 \times 100 \mu \mathrm{m}$ for the directions normal and transverse to rolling, and larger in the rolling direction.

Two types of measurements were made: first, the interplanar spacing of the $\{311\}$ plane family in the $x$ and $y$ directions was measured at points in a grid with a spatial resolution of $2.5 \mathrm{~mm}$ in the vicinity of the notch using a gauge volume of $2 \times 2 \times 2 \mathrm{~mm}$. The locations of measurements of this type are shown as red diamonds in Fig. 2. Second, strains at points in a finer grid $(1 \mathrm{~mm}$ spatial resolution, blue diamonds) around the tip of the notch were also measured using a gauge volume of 0.6 x $0.6 \times 2 \mathrm{~mm}$. At these points, the inter-planar spacing of the $\{311\}$ plane was measured in the $x$ and $z$ directions, while the spacing of the $\{222\}$ plane was measured for the $y$ direction. Due to the crystallographic texture of the specimen material it was necessary to use different planes in these different directions to achieve acceptable counting times with this smaller gauge volume. All of these measurements were compared with lattice parameter measurements taken from nominally un-stressed reference specimens in the same orientation, in order to calculate residual elastic strain. For the set of measurements using a gauge volume of $0.6 \times 0.6 \times 2 \mathrm{~mm}$ (in which residual elastic strains were measured in three orthogonal directions) residual stresses were calculated from the residual elastic strain data using hkl-specific elastic constants derived using the Kröner polycrystal modelling scheme [15, 16]. Finally, the $2 \times 2 \times 2 \mathrm{~mm}$ gauge volume was used to measure residual elastic strain in the $x$ direction only in a 'blank' specimen that had not undergone compression. This confirmed that the specimens were free of any measurable residual stress prior to indentation.

Using residual stress measurements from the indented specimen without a notch, a prediction of the residual stress contribution to the notched specimen stress intensity factors under perfectly elastic conditions was made via the method of weight functions. For this method, it is necessary to evaluate the residual stress which exists along the length of the prospective crack/notch in the un-cracked specimen. Although only two in-plane components of residual elastic strain were measured for the uncracked specimen, the results of finite element modelling of the punching process indicated that the stress in the out-of-plane direction in the un-cracked specimen was negligibly small along the prospective crack line. Therefore, the residual stress component in the cracktransverse direction $\left(\sigma_{y y}\right)$ could be estimated from the neutron diffraction measurements by assuming plane stress conditions. This is shown in Fig. 8. The weight function for a single-edge-notched specimen provided by Tada et al. [17] was then used to evaluate $K_{I}$.

Table 1 Number of different single-edge-notched bar specimens used in this study

\begin{tabular}{llll}
\hline & No notch & $7.5 \mathrm{~mm}$ notch & $15 \mathrm{~mm}$ notch \\
\hline Not indented & 1 & 0 & 8 \\
Indented & 1 & 1 & 8 \\
\hline
\end{tabular}


Fig. 2 Measurement locations used for the neutron diffraction measurements. (a.) Location of the measurement plane within a specimen. (b.) Locations of the measurement points relative to the notch tip

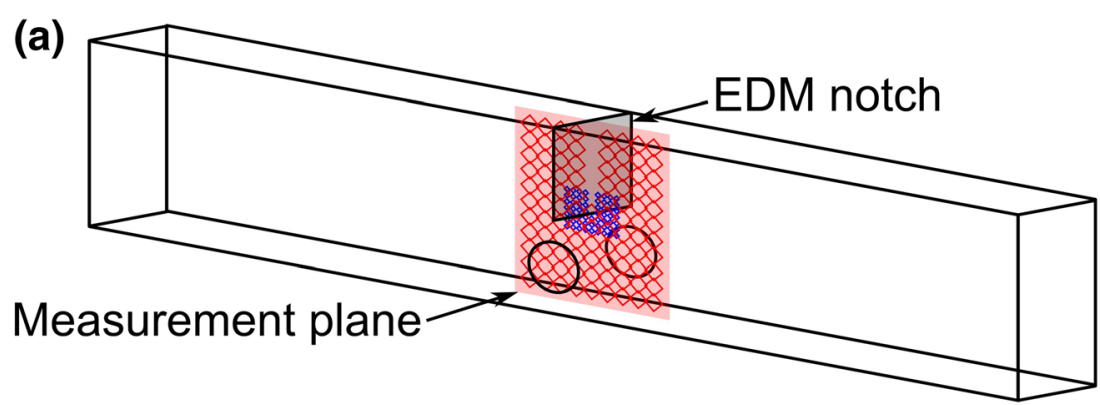

(b)

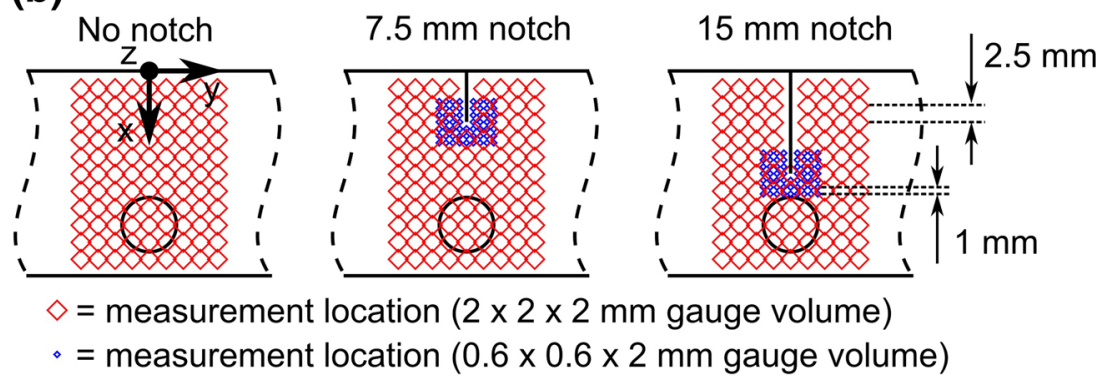

\section{Fracture Testing}

Three-point bend fracture tests were carried out using the specimens which contained $15 \mathrm{~mm}$ notches: eight of which were indented and eight non-indented (see Table 1). The specimens contained EDM-cut notches rather than sharp fatigue pre-cracks, but otherwise these tests were performed using the procedure described in ASTM E399-12e3 [18]. A support span of $120 \mathrm{~mm}$ and a loading rate of $0.5 \mathrm{~mm} / \mathrm{min}$ was used. Estimates of the stress intensity factor contribution at the notch tip due to the applied load were calculated assuming perfectly elastic conditions.

\section{Finite Element Simulation}

\section{Overview}

Elastic-plastic finite element analysis of the indented and non-indented specimens was performed. For the nonindented specimen (Model 1 in Fig. 3) it was only necessary to model the three-point bend loading, whereas for the indented specimen it was necessary to model the indentation process, cutting of the notch, and three-point bend loading in sequence (Model 2a). In addition to this, the indented specimen was analysed using a combined

\section{Non-indented specimen}

Model 1

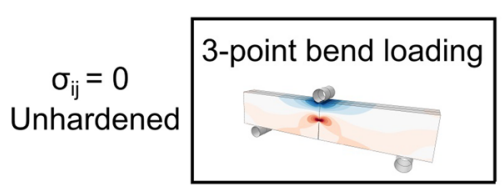

Indented specimen

Model 2a $\quad \sigma_{i j}=0$

Model 2a Unhardened
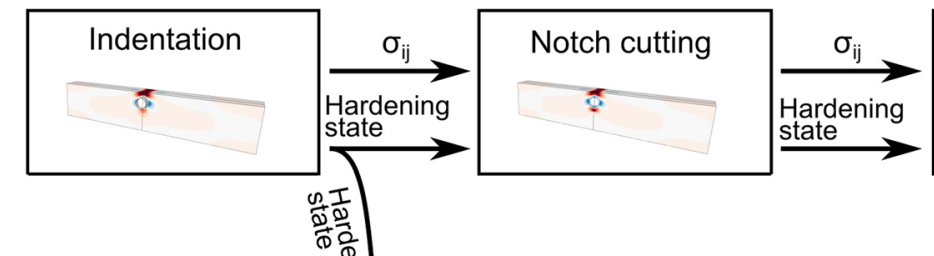

3-point bend loading

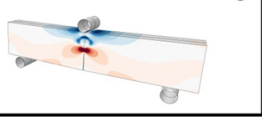

Model 2b
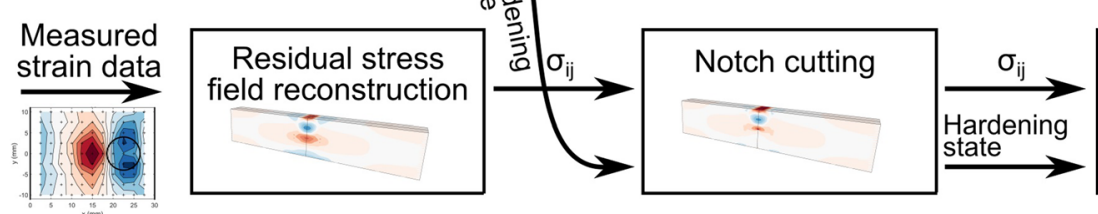

3-point bend loading

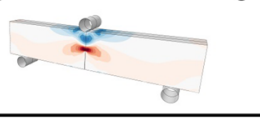

Fig. 3 The three finite element models used for simulating specimen indentation and three-point bend loading 
experimental/modelling approach (Model 2b). Residual elastic strain data measured using neutron diffraction was used to reconstruct the complete stress field in unnotched specimen. This was combined with material hardening state information taken from modelling of the indentation process to generate a model of the indented specimen, from which the notch cutting and three-point bend loading steps were then simulated.

\section{Conventional Modelling}

The indentation process, introduction of the notch, and the subsequent three-point bend loading were simulated using finite element analysis. The specimen material assumed to be approximately isotropic and to obey a von Mises yield law. Plastic deformation of the material was modelled using incremental flow plasticity. Non-linear strain-hardening of the material was modelled using the material's true stress-strain curve derived from the uniaxial tensile tests described in Appendix A, which was supplied to the FE solver in tabulated form (see Table 2). The material was assumed to obey an isotropic strainhardening behaviour. The material's mechanical properties were assumed to be rate-independent over the range of strain rates encountered and cyclic hardening effects were not considered. Since all of the specimens were symmetric about the plane containing the notch and about their mid-thickness only one quarter of the specimen was modelled, with appropriate boundary conditions imposed at the symmetry planes. The finite element mesh used to represent the quarter-specimen in the simulations contained 39,078 nodes and 34,560 8-noded reduced-integration linear brick elements, and is shown in Fig. 4. Additionally, 6-noded full-integration linear wedge elements were used at the crack tip. The Abaqus/Standard v6.12 finite element solver [19] was used for all of the calculations.

During simulation of the indentation process, the punch was modelled as a perfectly rigid cylinder and isotropic surface friction between the punch and specimen was imposed with a frictional coefficient of $\mu=0.5$,

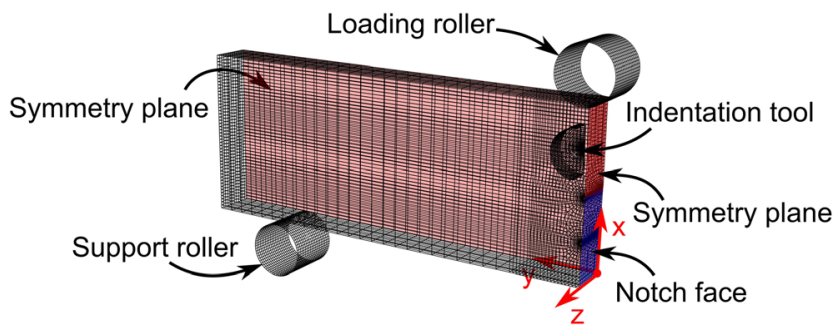

Fig. 4 Overview of the mesh used for finite element simulation of the indentation, notch introduction and three-point bend loading processes representative for this pair of materials $[20,21]$. The indentation tool was loaded using a vertical force equivalent to that used in the experiments: $37.5 \mathrm{kN}$ due to symmetry about the $\mathrm{x}-\mathrm{z}$ plane. Introduction of the notch was simulated by removing symmetric boundary conditions on the $\mathrm{x}-\mathrm{z}$ plane to the required notch length in four incremental steps. Throughout the analysis the notch was modelled as a sharp crack; the finite width of the notches in the real specimens was not considered. After crack introduction, the three-point bend loading of the specimens was simulated. As with the indentation process, the loading cylinders used in three-point bending were modelled as perfectly rigid cylinders, but their contact with the specimen was assumed to be frictionless. Values of the $J$-integral for the crack under incremental loading conditions were calculated from the stress and strain fields for each condition using the domain integral method [22]. For the indented specimens, it was necessary to use the modified form of the $J$-integral proposed by Lei $[8,23]$ to account for the effect of residual stress loading.

\section{Combined Experimental/Modelling Approach}

To avoid some of the difficulties involved in accurately simulating the introduction of residual stress, a technique for including measured residual stress data in the simulations was used in Model 2b (see Fig. 3). An estimate of the complete residual stress state in the un-notched specimens was reconstructed from measured residual elastic strain data using the iterative technique described previously by Coules et al. [24] and others [25, 26]. First, three

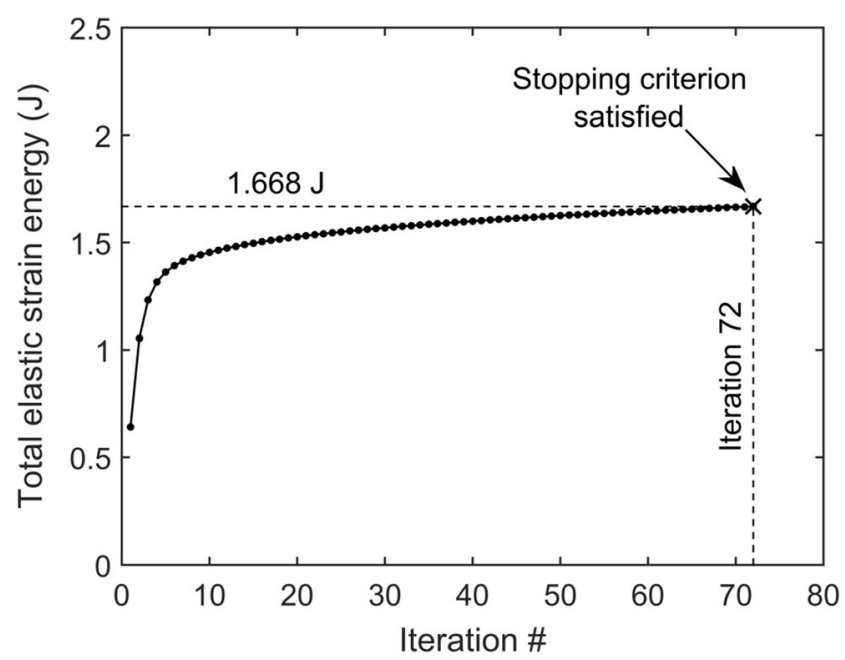

Fig. 5 Progress of the iterative residual stress field reconstruction for the indented specimens. The total elastic strain energy is calculated at the end of each iteration 
orthogonal components of the stress tensor at the neutron diffraction measurement locations were calculated assuming a state of plane stress. These were interpolated linearly in the $x-y$ plane over the region in which measurements were taken $(y \leq 10 \mathrm{~mm})$. The three components of the interpolated stress field in the measurement region were applied as an initial condition to a finite element model of the specimen. This stress state was then allowed to partially relax and establish a self-equilibrating stress field, using a facility for this purpose provided in the Abaqus/ Standard solver [19]. The measured stress components were then re-applied while the rest of the field was left unchanged, and the process was repeated iteratively until there was negligible change in the resulting stress field. The rate of change of the reconstructed stress field was estimated by calculating the total elastic strain energy at the end of each iteration. The iterative process was terminated when the difference in strain energy with the previous iteration was less than $0.1 \%$, which in this case took 72 iterations (see Fig. 5).

Material close to the indentation tool is strain-hardened during indentation. However, the hardening state of the material can be predicted far more accurately using finite element analysis than the residual stress field because it
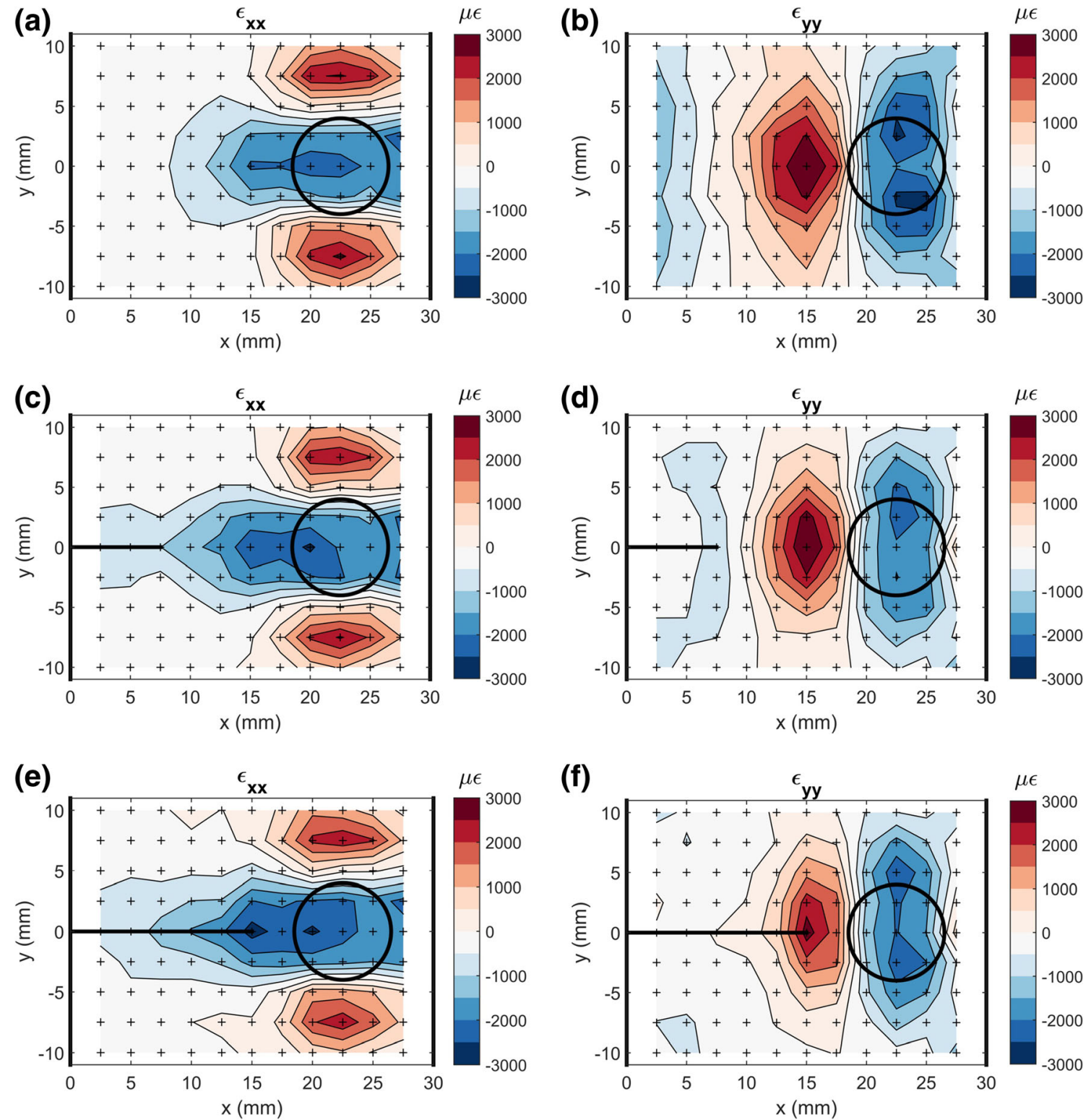

Fig. 6 Residual elastic strain measured using neutron diffraction at the mid-thickness plane of the specimens in the region of the notch: (a,b.) specimen with no notch, (c,d.) specimen with $7.5 \mathrm{~mm}$ notch, (e,f.) specimen with $15 \mathrm{~mm}$ notch. Crosses indicate diffraction gauge volume centres 

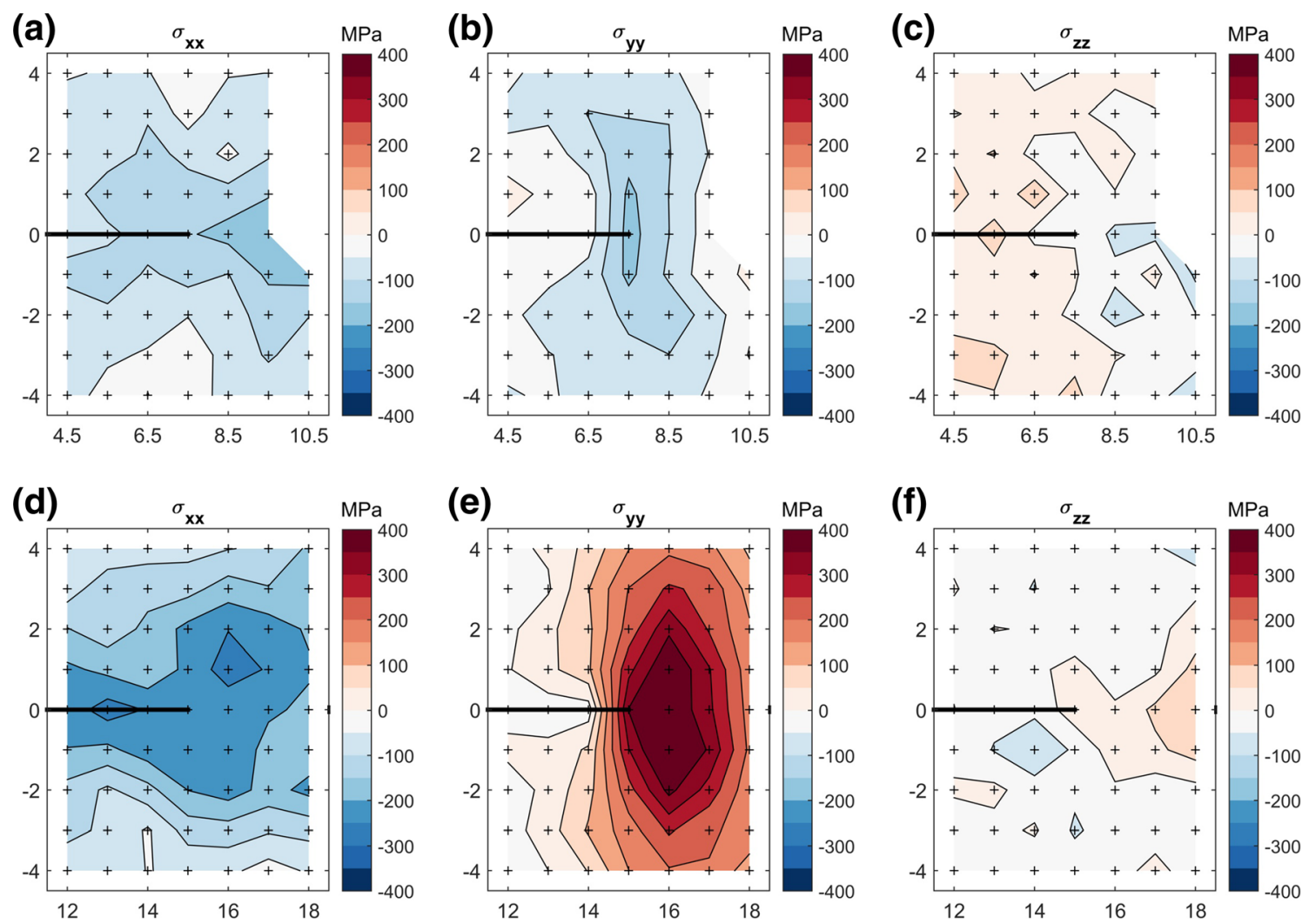

Fig. 7 Residual stress around the notch tip measured at the mid-thickness of the specimen: (a-c.) $7.5 \mathrm{~mm}$ notch, $(\mathbf{d}-\mathbf{f}$.) $15 \mathrm{~mm}$ notch. Diffraction gauge volume centroids are indicated by ' + '. The missing area of the stress map in $(\mathbf{a}-\mathbf{c})$ is caused by an incomplete measurement

develops during the compression part of the indentation process and is not affected by unloading as the indentation tool is raised. It can therefore be predicted accurately using only monotonic material stress-strain data. Here, the hardening state of the material was taken from the existing model of the indentation process (see Fig. 3). The reconstructed residual stress field and the material hardening state were applied as initial conditions prior to a simulation of notch cutting and bend loading.

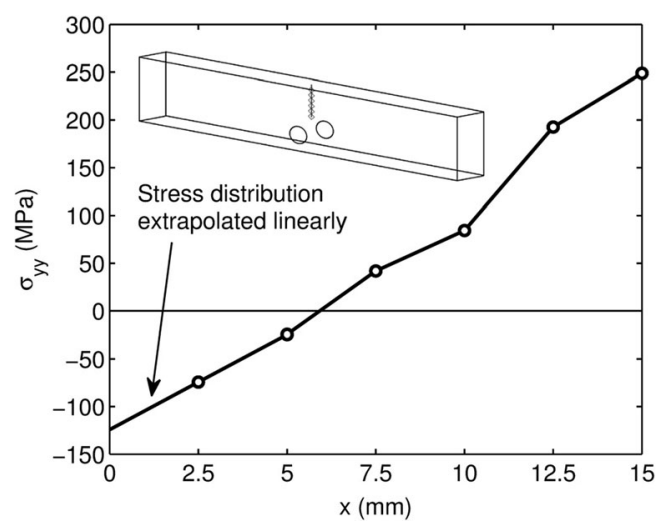

Fig. 8 Distribution of residual stress in the transverse direction $\left(\sigma_{\mathrm{yy}}\right)$ along the prospective notch line at the mid-plane of an indented but unnotched specimen. Stress calculated assuming plane stress conditions

\section{Results}

\section{Measured Residual Elastic Strain and Stress Fields}

All results are given in the coordinate system shown in Fig. 2(b) and Fig. 4. Figure 6 shows the distribution of residual elastic strain at the mid-plane of the bar specimens in the region of the notch. Incremental extension of the notch causes the residual stress field throughout the measured region to partially relax while a concentration in residual stress arises at the notch tip. For these measurements, the contribution of diffraction peak fitting uncertainty to the measurement error was evaluated using formulae provided by Wimpory et al. [27] to be approximately $38 \mu \varepsilon$ for the $x$ direction and $57 \mu \varepsilon$ for the $y$ direction. The strain error due to other sources of measurement uncertainty was not evaluated.

Maps of the residual stress field at higher resolution around the notch tip for 7.5 and $15 \mathrm{~mm}$ notches are shown in Fig. 7. The plots of stress in the notch-transverse direction $\left(\sigma_{y y}\right.$, Fig. 7(b) \& (e)) show that the residual stress field around a notch of $7.5 \mathrm{~mm}$ favours notch closure, while the tensile stresses ahead of the $15 \mathrm{~mm}$ crack favour notch opening.

Mode I stress intensity factors were determined from the residual elastic strain maps of the un-notched specimen (Fig. 6(a) \& (b)) using the weight function method described in Section 2.3. Plane stress conditions along the 
Fig. 9 Results of three-point bend fracture testing of specimens containing $15 \mathrm{~mm}$ EDM notches, with and without precompression (8 specimens of each). (a.) Measured load/crack mouth opening displacement curves. (b.) Cumulative probability of fracture as a function of applied stress intensity (a)

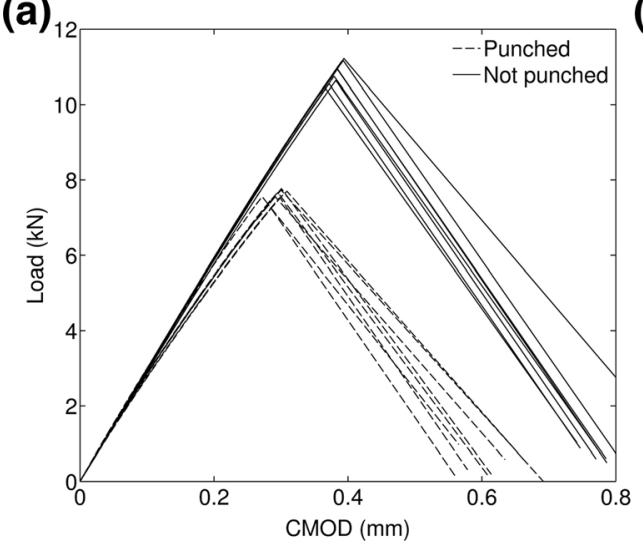

(b)

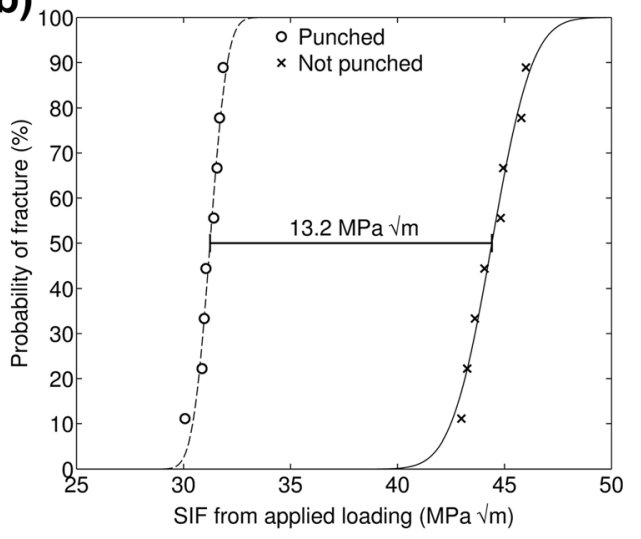

prospective notch line prior to notch introduction were assumed, allowing the notch-line stress distribution shown in Fig. 8 to be calculated. The stress intensity factors calculated from this stress distribution were: $-11.6 \mathrm{MPa}$ $\sqrt{\mathrm{m}}$ for a $7.5 \mathrm{~mm}$ notch and $+11.3 \mathrm{MPa} \sqrt{\mathrm{m}}_{\mathrm{m}}$ for a $15 \mathrm{~mm}$ notch. The negative stress intensity factor calculated for the $7.5 \mathrm{~mm}$ notch implies crack closure, but since the notch had a finite width (approximately $165 \mu \mathrm{m}$ ) no contact between the notch faces was observed in any specimen.

\section{Fracture Test Results}

In all of the three-point bend tests performed, the specimens were observed to fracture in an abrupt and apparently brittle manner. Load/CMOD curves for all 16 specimens are shown in Fig. 9(a): the pre-compression process has reduced the loadbearing capacity of the specimens. Using this data, the cumulative probability of failure for each set of specimens was calculated according to [28]:

$P(F)=\frac{n(F)}{N+1}$

Where $n(F)$ is the number of specimens failed at loading force $F$ from a total of $N$ specimens, and $P$ is the cumulative probability of failure. The apparent stress intensity factor for the notch at fracture was calculated using equations provided in
ASTM E399-12e3 [18], which for this specimen geometry and loading mode reduce to:

$K_{I}=4.099 \mathrm{~F}$

However, there are two factors which affect the validity of stress intensity factor results derived in this way. Firstly, the specimens were not fatigue pre-cracked and so fracture initiated from the tip of a relatively blunt notch rather than a sharp crack tip. Secondly, the apparent fracture toughness values were slightly beyond those allowable for a specimen of this thickness and yield stress according to ASTM E399-12e3. The cumulative probability of fracture is plotted against apparent applied Mode I SIF in Fig. 9(b). On average there is a reduction of $13.2 \mathrm{MPa} \sqrt{\mathrm{m}}_{\mathrm{m}}$ the apparent stress intensity due to applied load at fracture for the punched specimens with respect to non-punched specimens.

The surfaces of fractured specimens were examined using scanning electron microscopy and characteristic micrographs are shown in Figure 10. Fracture has occurred via a combination of grain boundary separation with some dimpled rupture, resulting in a ridged fracture surface on which grain outlines are clearly visible. The fracture surface is largely homogeneous across the specimen thickness with no visible evidence of through-thickness variation in the mechanism of fracture, although shear lips with a depth of around $1 \mathrm{~mm}$ occur at each surface. No difference between the fracture surfaces of indented and nonindented specimens was observed.
Figure 10 Fracture surface of a non-indented specimen. a.) Macrograph of the complete specimen cross-section, b.) notch tip at 100x magnification, c.) fracture surface at $1000 \mathrm{x}$ magnification. SEM images taken at $15 \mathrm{keV}$; combination of backscattered and secondary electron signals (a)

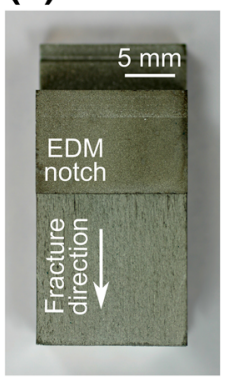

(b)

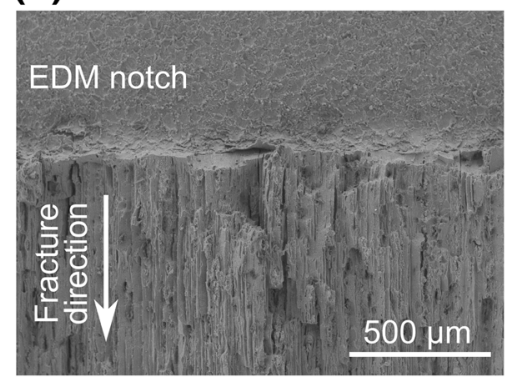

(c)

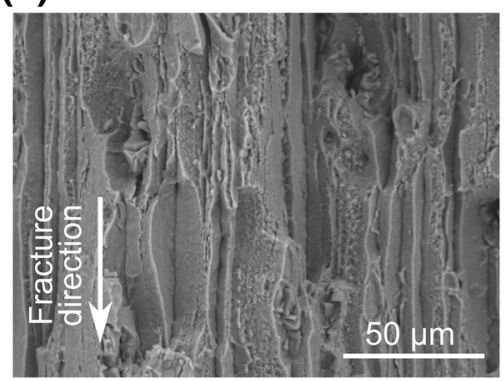


Fig. 11 Residual elastic strain field following indentation, as predicted using elastic-plastic FEA of the indentation process $(\mathbf{a}, \mathbf{b}.) \varepsilon_{\mathrm{xx}}$ and $\varepsilon_{\mathrm{yy}}$ at the midthickness (i.e. $\mathrm{z}=0$ plane) of the specimen, (c.) overview showing the $\varepsilon_{\mathrm{yy}}$ component for the complete specimen
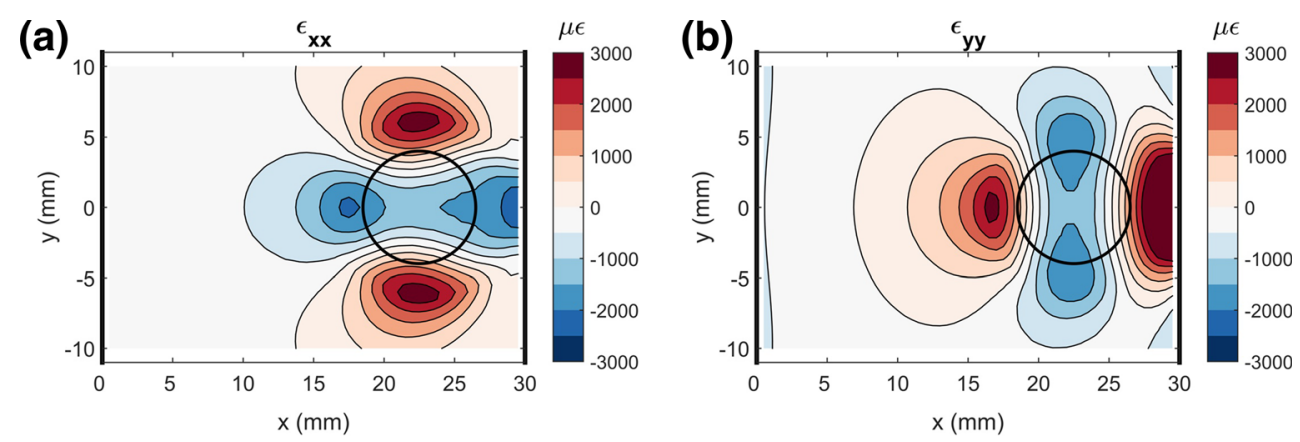

(c)

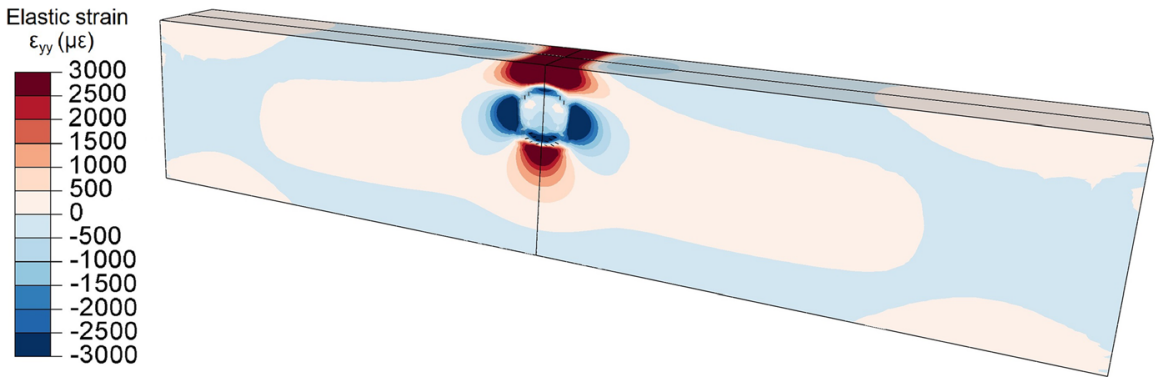

\section{Finite Element Simulation Results}

As described in Section 3.2, finite element analysis of the indentation process was used to predict the distribution of residual elastic strain in an indented specimen (Model 2a). This prediction is shown in Fig. 11. Comparing this to the neutron diffraction results for the specimen mid-plane presented in Fig. 6(a) \& (b) there is general qualitative agreement, but the predicted field is more intense than that observed in the measurements. This may be a consequence of the inelastic material properties used in the model: the only basic hardening properties have been defined using the available half-cycle uniaxial test data, whereas a strain reversal occurs some parts of the specimen during indentation.

The magnitude of plastic deformation which occurs during compression was calculated using Model 2a, and is shown in Fig. 12. Although the indentation process produces large plastic strains in material directly beneath the compression tool, no

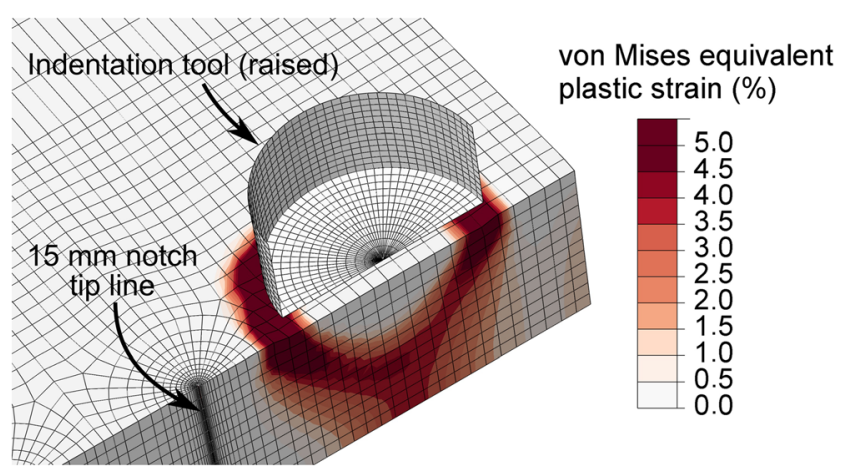

Fig. 12 Plastic strain in the region of the indentation tool following indentation, according to Model $2 \mathrm{a}$ plasticity occurs in the region where the $15 \mathrm{~mm}$ notch tip is subsequently introduced. Therefore no work-hardening of the notch tip material, which could change its apparent fracture initiation properties, occurs during indentation.

Reconstruction of the residual stress field in the indented specimen from the neutron diffraction data yielded a slightly different distribution of residual stress to the one calculated by Model 2a. Fig. 13 shows the distribution of residual elastic strain according to the reconstruction. This agrees well with the data from which it was reconstructed, as shown in Fig. 14(a). Model 2b, which uses this reconstructed field as input, also produces results which continue to show good agreement with the measured data as the notch is incrementally introduced (see Fig. 14(b) \&(c)). Overall, the Model 2b approach of measurement, residual stress field reconstruction and then modelling of notch introduction/loading gave better agreement with experimental strain data than the Model 2a method (i.e. modelling the indentation process).

The $J$-integral at fracture was evaluated from the results of each of the three models using fracture loads determined experimentally. Good path-independence of $J$ was observed beyond $1 \mathrm{~mm}$ radius from the notch tip (see Fig. 15(a)) and results evaluated using a circular domain of radius $3 \mathrm{~mm}$ were taken to be reliable. The $J$-integral at fracture is shown in Fig. 15(b) as a function of the through-thickness dimension $z$. Assuming that the $J$-integral is a reliable criterion for fracture initiation in these specimens then the maximum value of $J$ at fracture should be the same for specimens with and without indentation. The distribution of $J$ at fracture calculated using a residual stress field reconstructed from measurements (Model 2b) agrees very well with the result for an un-indented specimen. The residual stress field calculated in Model 2a was different from that observed in 
Fig. 13 Residual elastic strain field following indentation, as reconstructed using the neutron diffraction measurements shown in Fig. $6\left(\right.$ a) $\&$ (b). (a, b.) $\varepsilon_{\mathrm{xx}}$ and $\varepsilon_{\mathrm{yy}}$ at the mid-thickness (i.e. $\mathrm{z}=0$ plane) of the specimen, (c.) overview showing the $\varepsilon_{\mathrm{yy}}$ component for the complete specimen
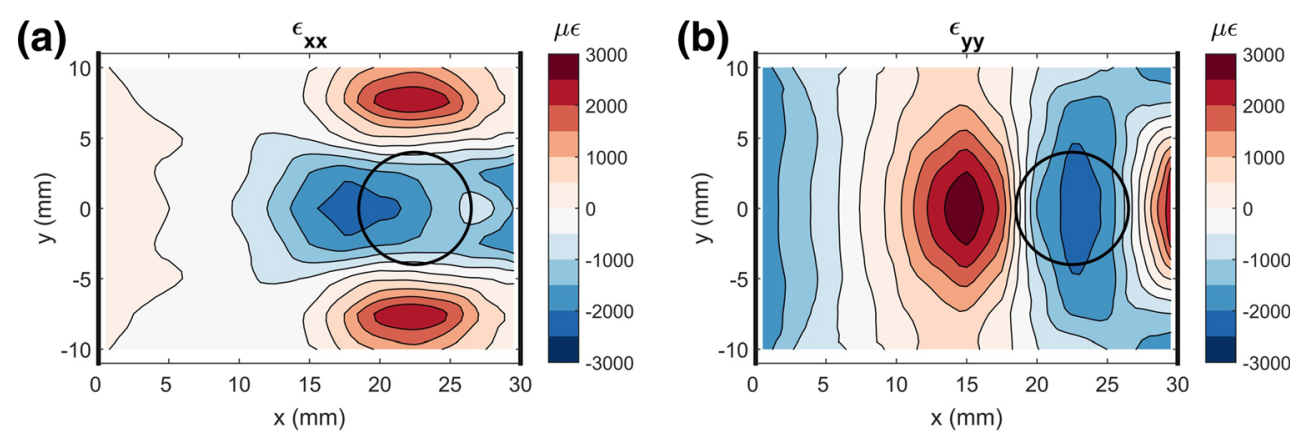

(c)

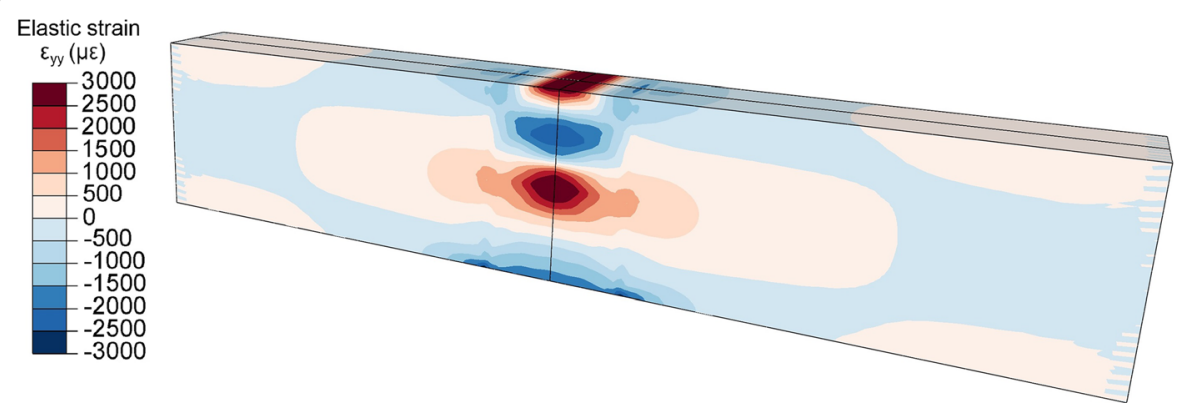

the real specimens, and this model predicts significantly higher $J$ values at the experimentally-determined fracture load.

\section{Discussion}

\section{Specimen Behaviour}

In Section 4.1, weight function analysis using neutron diffraction measurements of an un-notched specimen was applied to predict the contribution of residual stress to the Mode I stress intensity factor at the specimen mid-thickness: $11.3 \mathrm{MPa} \sqrt{ } \mathrm{m}$. This is similar to the reduction in apparent fracture toughness of the residually-stressed specimens determined using fracture tests (13.2 MPa $\sqrt{\mathrm{m}}$, see Fig. 9(b)). This suggests that only limited plasticity occurs during notch introduction and threepoint bend loading so that for these specimens, the effects of residual and applied loading on the specimen's proximity to fracture are almost perfectly additive. Widespread plasticity would allow stress relaxation which would cause the initial
Fig. 14 Comparison of the measured and reconstructed elastic strain distributions $\left(\varepsilon_{\mathrm{yy}}\right.$ component shown) at the midplane of the specimen. (a.) Reconstructed elastic strain distribution in the un-notched specimen. (b,c.) Incremental introduction of the notch to 7.5 and $15 \mathrm{~mm}$ respectively, simulated using Model $2 \mathrm{~b}$
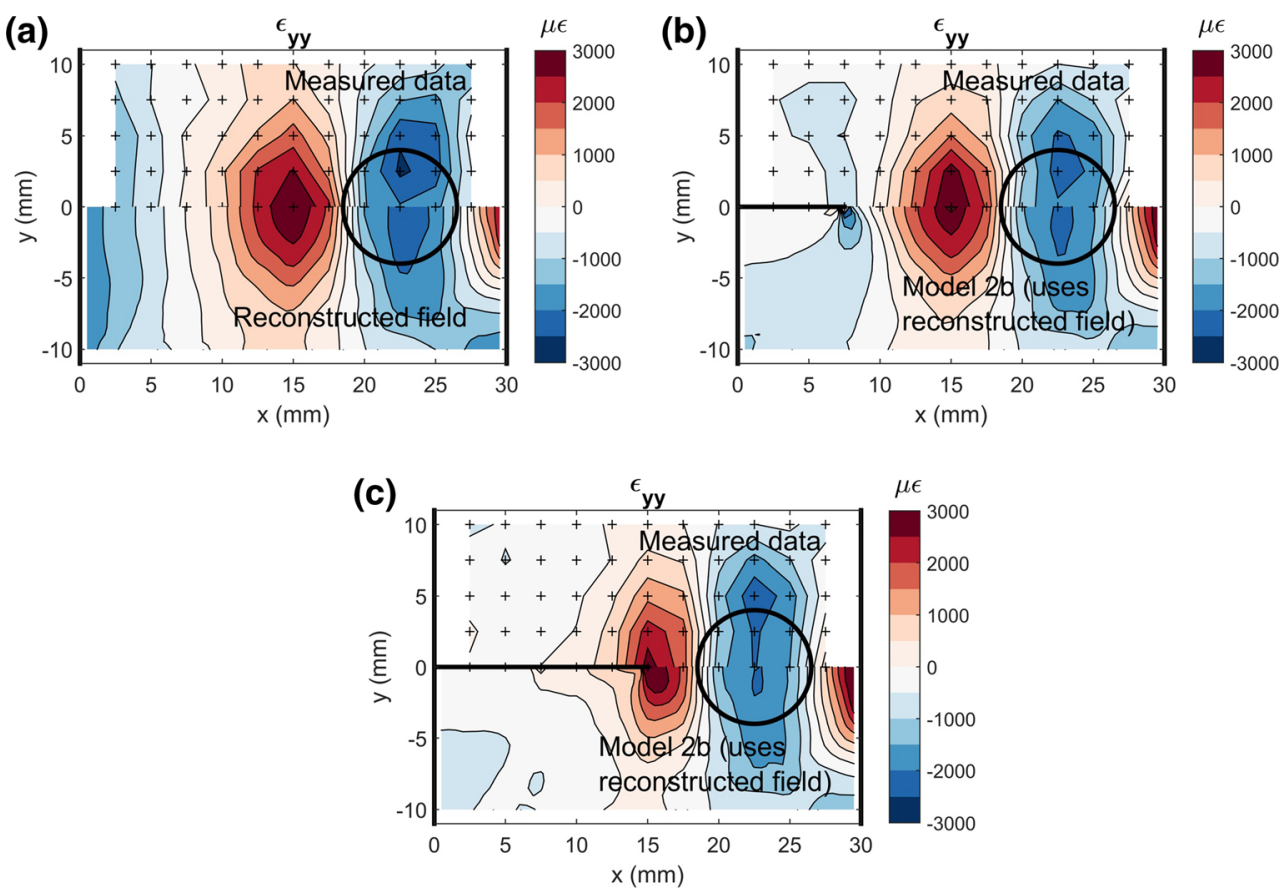

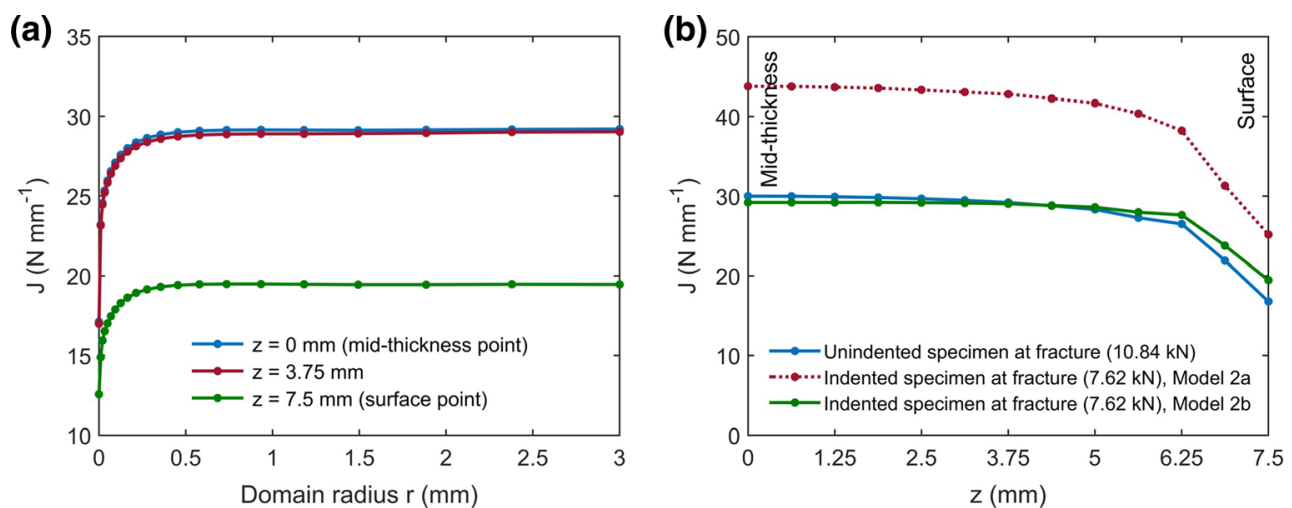

Fig. $15 \mathrm{~J}$-integral results determined from the finite element simulations. (a.) Example showing $\mathrm{J}$ evaluated over different domains at three points on the notch tip line in Model $2 \mathrm{~b}$ with an applied load of $7.62 \mathrm{kN}$, (b.) J -integral at fracture as a function of through-thickness dimension $\mathrm{z}$ for indented and nonindented specimens. The fracture loads were determined from the experiments (Fig. 9(a))

residual stress state to have a diminished influence on apparent fracture toughness. The elastic-plastic crack driving force can be expressed as an elastic-plastic equivalent stress intensity factor $K_{J}$ :

$K_{J}=\sqrt{J \frac{E}{1-\nu^{2}}}$

where $E$ is Young's modulus and $\nu$ is Poisson's ratio. Fig. 16 shows $K_{J}$ at the mid-thickness of the specimen (the most severe condition on the notch tip line) against the applied load. This illustrates the largely elastic nature of the fracture process in these specimens. In both cases there is an almost linear relationship between the equivalent stress intensity factor and the applied load, and the $K_{J}$ only deviates slightly from the Mode I SIF calculated for a perfectly elastic material (dashed lines). The load-CMOD curves shown in Fig. 9 and the fractographs in Figure 10 provide further evidence that fracture occurred in a largely brittle manner.

The specimens used in this work contained EDM-cut notches that were not fatigue pre-cracked prior to fracture loading. This ensured that the crack front was the same shape and in the same position for all specimens, whereas fatigue pre-cracking in the presence of residual stress can lead to a non-uniform crack front shape [29, 30]. This method worked well for the purpose of comparing two sets of specimens, but it is important to note that the values of SIF calculated from the fracture test results are only 'apparent' values and cannot be taken to be representative of the material's plane strain fracture toughness.

\section{Calculation of Fracture Parameters Using Measured Residual Stresses}

Although fracture occurred in a largely brittle manner, the contribution of the measured residual stress field to the elastic-plastic crack driving force was also studied. The notch tip through-thickness $J$ distribution at fracture in the indented specimens was calculated using measured residual stress data in conjunction with a residual stress field reconstruction technique, and it corresponds closely to the distribution for an unindented specimen at fracture (Fig. 15). This capability for calculating elastic-plastic fracture parameters using measured data could also be used to study the interaction between residual and applied stress during more ductile fracture.
Fig. 16 Elastic-plastic stress intensity factor $\mathrm{K}_{\mathrm{J}}$ at the midthickness of the specimens during three-point bend loading. The Mode I SIF calculated for perfectly elastic conditions is shown for comparison. Contour plots show the von Mises equivalent plastic strain around the crack tip on the specimen mid-plane

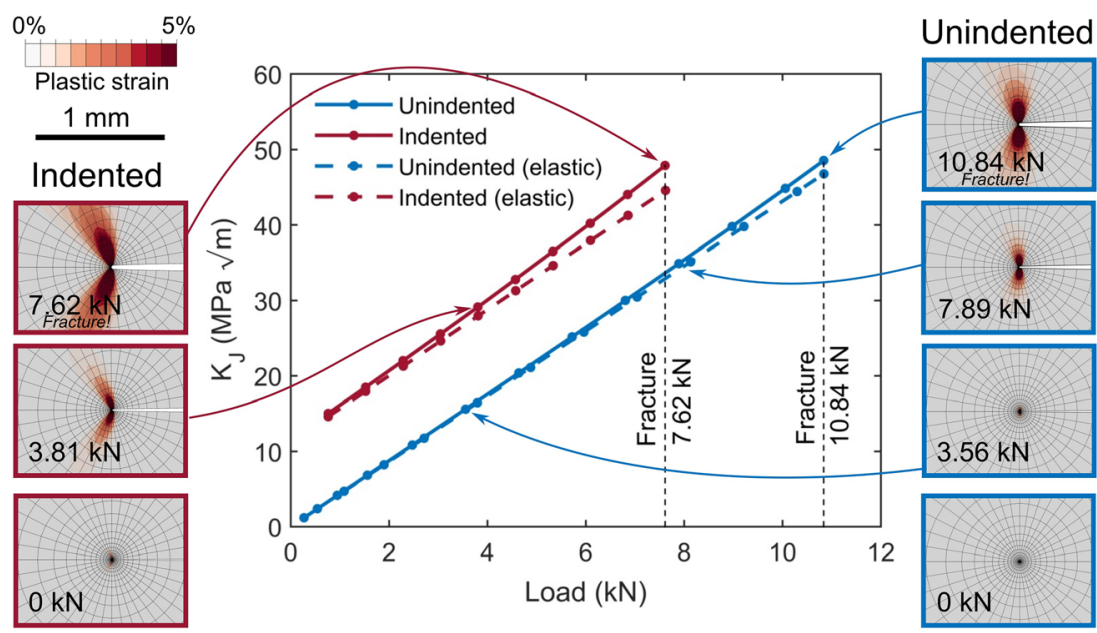


Typically, when measured residual stress data is incorporated into the analysis of elastic-plastic fracture, it is via simplified methods such as the Failure Assessment Diagram (FAD) approach used in R6 and BS 7910:2013. In general, to calculate the elastic-plastic crack driving force explicitly it is necessary to have accurate estimates of the stress and strain fields surrounding the crack tip. However, residual stresses present within a component prior to fracture loading affect the subsequent development of strain, so for calculation of the energy release rate a detailed characterisation of any pre-existing residual stress field is needed. For a crack front in a three-dimensional component or specimen, the level of residual stress field characterisation required for this is currently beyond the capability of most measurement techniques. Here, we have demonstrated that a method for reconstructing a residual a stress field from limited measurements can be used in conjunction with finite element analysis in order to enable the evaluation of crack parameters in the presence of residual stress via the well-known contour integral method. The reconstructed residual elastic strain field and the elastic strain fields calculated in subsequent modelling steps agreed closely with neutron diffraction results. Using $J$ integral results calculated in this way it would be possible to perform much more detailed investigations into the effects of residual stress on fracture. For many processes that cause residual stresses in structural components, residual stress prediction via FEA is particularly challenging and may require extensive experimental validation. The use of measured residual stress results in conjunction with modelled or measured material hardening state data therefore represents an attractive alternative method.

\section{Limitations}

There are two significant limitations to the method of stress field reconstruction used in Model $2 \mathrm{~b}$. Firstly, a relatively large quantity of measured data is required to reconstruct the residual stress field surrounding the crack accurately. The data requirement for reconstruction is discussed by Coules et al. [24]. In this study, we used neutron diffraction to measure the residual stress field, resulting in a measured 2D strain map at reasonably high spatial resolution of $2.5 \mathrm{~mm}$ (Fig. 6(a) \& (b)). However, many other methods of residual stress measurement are more limited in terms of resolution, measurement depth, and the number of strain components, which would make it difficult to generate the quantity of data required for stress field reconstruction. Secondly, for analysis of the crack with applied load, the material hardening state prior to crack introduction is needed in addition to the residual stress field. Here, we used modelling of the indentation process to generate a hardening state map, but for some processes such as welding this would be more complicated. In such cases it might be more convenient to determine the material hardening state using measurements.

\section{Conclusions}

In this work we have demonstrated a method for incorporating measured residual elastic strain or stress data into a finite element simulation of elastic-plastic fracture. This technique allows measured data to be included in an elastic-plastic analysis without the use of simplified methods such as the failure assessment diagram approach. A simulation of a specimen containing a residual stress field reconstructed from neutron diffraction data showed good agreement with measured data corresponding to later modelling steps, and was consistent with experimental fracture test results. In the example presented in this study, all of the fracture specimens failed in a largely brittle manner and residual stress had a substantial effect on the apparent fracture toughness. However the technique we have proposed could also be applied to study more ductile fractures in which the effects of primary and secondary loading superimpose in a more clearly non-linear manner.

Acknowledgments This work was supported by a Royal Academy of Engineering/Rolls Royce/EDF Energy chair held by the late Prof. David Smith. Access to the SALSA diffractometer was provided by the Institut Laue-Langevin under proposal no. 1-02-148.

\section{Appendix A: Mechanical properties of aluminium alloy 7075-T6 specimen material}

Uniaxial tensile tests at ambient temperature to BS EN ISO 6892-1:2009 [31] were performed on the specimen material to determine its mechanical properties. The cylindrical test specimens had a diameter of $5 \mathrm{~mm}$ and a parallel length of $30 \mathrm{~mm}$ oriented in the direction transverse to rolling. A representative stress/strain curve for the material derived from the results of these tests is shown in Fig. 17.

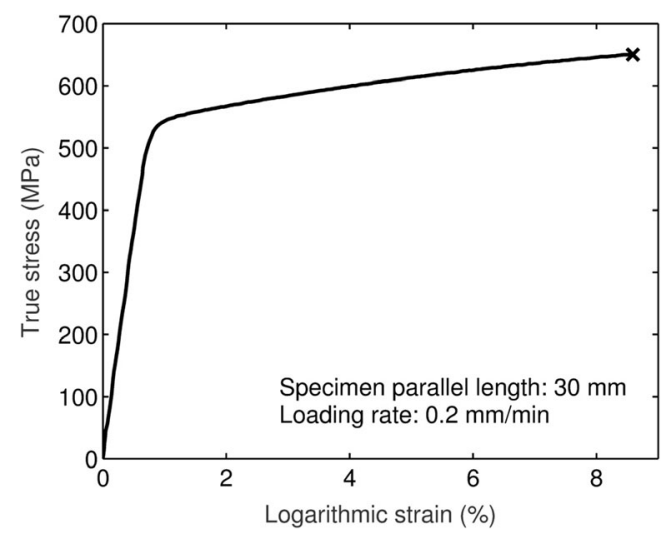

Fig. 17 Representative uniaxial stress/strain curve for aluminium alloy 7075-T6 in the direction transverse to rolling 
The Young's modulus was determined to be $69.8 \mathrm{GPa}$ and true stress vs. logarithmic plastic strain data for the material are given in Table 2. The Poisson's ratio of the material was not evaluated experimentally but was assumed to be equal to 0.33 in the subsequent modelling work.

Table 2 Flow stress-plastic strain data for aluminium alloy 7075-T6 in the direction transverse to rolling

\begin{tabular}{ll}
\hline True stress $(\mathrm{MPa})$ & Logarithmic plastic strain (\%) \\
\hline 486.1 & 0 \\
527.2 & 0.0944 \\
543.4 & 0.317 \\
560.8 & 1.0 \\
578.9 & 2.0 \\
609.3 & 4.0 \\
632.7 & 6.0 \\
651.5 & 8.0 \\
664.3 & 10.0 \\
\hline
\end{tabular}

Open Access This article is distributed under the terms of the Creative Commons Attribution 4.0 International License (http:// creativecommons.org/licenses/by/4.0/), which permits unrestricted use, distribution, and reproduction in any medium, provided you give appropriate credit to the original author(s) and the source, provide a link to the Creative Commons license, and indicate if changes were made.

\section{References}

1. Bueckner HF (1973) "Field singularities and related integral representations". Methods of analysis and solutions of crack problems, vol. 1, G. C. Sih, Ed. Noordhoff International, 239-314

2. Budden PJ, Sharples JK (2003) "Treatment of secondary stresses". Comprehensive Struct Integrity, vol. 7, 1st ed., R. A. Ainsworth and K.-H. Schwalbe, Eds. Elsevier-Pergamon, 245-288

3. Ainsworth RA, Hooton DG (2008) R6 and R5 procedures: the way forward. Int J Press Vessel Pip 85(3):175-182

4. EDF Energy, Gloucester (2013) R6: Assessment of the integrity of structures containing defects, revision 4, Amendment 10

5. BSi (2013) BS 7910:2013 - Guide to methods for assessing the acceptability of flaws in metallic structures

6. Ainsworth RA, Sharples JK, Smith SD (2000) Effects of residual stresses on fracture behaviour - experimental results and assessment methods. J Strain Anal Eng Des 35(4):307-316

7. Ainsworth RE, Neale BK, Price RH (1978) "Fracture behaviour in the presence of thermal strains". Proc Conf Tolerance Flaws Pressurised Components IMechE C96/78

8. Lei Y (2005) J-integral evaluation for cases involving nonproportional stressing. Eng Fract Mech 72(4):577-596

9. Wilson WK, Yu I-W (1979) The use of the J-integral in thermal stress crack problems. Int J Fract 15(4):377-387
10. Lindgren L-E (2007) Computational welding mechanics. Woodhead Publishing,

11. Mahmoudi AH, Truman CE, Smith DJ (2008) Using local out-of-plane compression (LOPC) to study the effects of residual stress on apparent fracture toughness. Eng Fract Mech 75(13):1516-1534

12. Pirling T, Bruno G, Withers PJ (2006) SALSA - a new instrument for strain imaging in engineering materials and components. Mater Sci Eng A 437(1):139-144

13. Holden TM (2013) "Neutron diffraction". practical residual stress measurement methods, 1st ed., G. S. Schajer, Ed. Wiley

14. Krawitz AD (2011) Neutron strain measurement. Mater Sci Technol 27(3):589-603

15. Hutchings MT, Withers PJ, Holden TM, Lorentzen T (2005) Introduction to the characterization of residual stress by neutron diffraction. Taylor and Francis

16. Kröner E (1958) Berechnung der elastischen Konstanten des Vielkristalls aus den Konstanten des Einkristalls. Z Phys 151(4): 504-518

17. Tada H, Paris PC, Irwin GR (2000) The stress analysis of cracks handbook, 3rd ed. Professional Engineering Publishing

18. ASTM International (2009) ASTM, E399-09 Standard test method for linear-elastic plane-strain fracture toughness KIc of metallic materials, no. E399-09

19. Abaqus/Standard v6.12. Providence, RI, USA: Dassault Systemes Simulia Corp., 2012.

20. Hutchings IM (1992) Tribology: friction and wear of engineering materials. CRC Press

21. Avallone EA, Baumeister T. Eds. (1996) Marks' standard handbook for mechanical engineers, 10th ed. McGraw-Hill

22. Shih CF, Moran B, Nakamura T (1986) Energy release rate along a three-dimensional crack front in a thermally stressed body. Int $\mathrm{J}$ Fract 30(2):79-102

23. Lei Y (2015) "J calculation for a crack in a welding residual stress field following a FE welding simulation". Trans SMiRT 23 Manchester, United Kingdom 213

24. Coules HE, Smith DJ, Abburi Venkata K, Truman CE (2014) A method for reconstruction of residual stress fields from measurements made in an incompatible region. Int J Solids Struct 51(10): 1980-1990

25. Do SC (2014) "Role of residual stress relaxation in fracture of stainless steel," University of Bristol

26. Serasli K, Coules H, Smith D (2015) "Residual stresses in clad nuclear reactor pressure vessel steels; prediction, measurement and reconstruction”. Proc ASME 2015 Pressure Vessels Piping Conf 2015-45224

27. Wimpory RC, Ohms C, Hofmann M, Schneider R, Youtsos AG (2009) Statistical analysis of residual stress determinations using neutron diffraction. Int J Press Vessel Pip 86(1):48-62

28. Khalili A, Kromp K (1991) Statistical properties of Weibull estimators. J Mater Sci 26(24):6741-6752

29. James P, Ford M (2014) "STYLE: modelling of the extraction of bend specimens from repair weld and the influence of residual stress on fracture testing”. Am Soc Mech Eng, Pressure Vessels Piping Div (Publ) PVP 6A:28395

30. Towers O, Dawes MG (1985) "Welding Institute Research on the Fatigue Precracking of Fracture Toughness Specimens". Elasticplastic fracture test methods: the user's experience, E. T. Wessel and F. J. Loss, Eds. ASTM, 23-46

31. BSi (2009) BSi, 6892-1:2009 Metallic materials - tensile testing part 1: method of test at ambient temperature 